\title{
Students' choices when solving expectation value problems
}

\author{
Gina Passante, ${ }^{1}$ Homeyra Sadaghiani, ${ }^{2}$ Steven J. Pollock, ${ }^{3}$ and Benjamin P. Schermerhorn ${ }^{1,2}$ \\ ${ }^{1}$ Department of Physics, California State University Fullerton, 800 N. State College Blvd., Fullerton, CA, 92831 \\ ${ }^{2}$ Department of Physics and Astronomy, California State Polytechnic University, $3801 \mathrm{~W}$. Temple Ave, Pomona CA, 91768 \\ ${ }^{3}$ Department of Physics, University of Colorado, 390 UCB, Boulder, CO, 80309
}

\begin{abstract}
In quantum mechanics, one is often interested in the expectation value (or average) of different quantities. It is possible to find expectation values for many different physical systems, for example the energy or position of a particle in the infinite square well, the $z$-component of spin for an electron, or the angular momentum of an electron in a hydrogen atom. In addition to the different physical contexts where the expectation value can be calculated, there are several methods one can use to solve for the expectation value. Depending on the information given in the problem, it might be easiest to solve using an integral, matrix mathematics, or a finite summation. In this work we analyze student responses to expectation value exam questions in several different physical contexts from several institutions, with a goal of determining which calculation methods and representations students choose most frequently and if some methods are more likely to result in correct answers.
\end{abstract}

\section{INTRODUCTION}

Unlike in classical mechanics, quantum mechanical systems often do not have a deterministic value for a given observable as the system can be in a superposition state. As such, a common activity in an undergraduate quantum mechanics course is to calculate the expectation value of a given state. The expectation value is the average value that would be measured if you had many identical copies of the quantum state and measured them all. Expectation values are a particularly rich concept for study given their application to any relevant observable in many different contexts. For example, one can ask about the expectation value of energy for a particle in an infinite square well, or the expectation value of spin in the $x$-direction for a spin-1/2 particle.

Additionally, it is possible to calculate the expectation value in several different ways. Three of the most common methods used in an introductory quantum mechanics course are what we call integration, matrix, and summation. The most generic equation for the expectation value for operator $\hat{A}$ is written in Dirac notation for pure states as $\langle\hat{A}\rangle=\langle\psi|\hat{A}| \psi\rangle$. This expression can be evaluated several different ways. When the state and operator are written as functions, for example, of $x$, it can be evaluated using integration as seen in Equation (1).

$$
\langle\hat{A}\rangle=\langle\psi|\hat{A}| \psi\rangle=\int \psi(x)^{*} \hat{A} \psi(x) d x
$$

The same expression can be evaluated using matrices for finite, discrete systems, such as a spin-1/2 particle. In this case the state of the system $|\psi\rangle$ can be represented as a twodimensional vector and the operator is a two-by-two matrix, as seen in Equation (2).

$$
\langle\hat{A}\rangle=\langle\psi|\hat{A}| \psi\rangle=\left(\begin{array}{ll}
a^{*} & b^{*}
\end{array}\right)\left(\begin{array}{ll}
A_{11} & A_{12} \\
A_{21} & A_{22}
\end{array}\right)\left(\begin{array}{c}
a \\
b
\end{array}\right)
$$

Finally, in this paper we consider a third method for evaluating the expectation value as an average of the possible measurement outcomes weighted by their respective probabilities. This summation is shown in Equation (3), where $p_{i}$ is the probability of outcome $a_{i}$.

$$
\langle\hat{A}\rangle=\langle\psi|\hat{A}| \psi\rangle=\sum_{i} p_{i} a_{i}
$$

Each of these methods have their advantages and drawbacks. In some questions only one method is viable, whereas in others, multiple methods can be used. These three methods are closely tied to three different notations that can be used to represent quantum states: wave functions, matrix notation, and Dirac notation. Gire and Price categorized the structural features of each notation [1]. They found that the students interviewed needed prompting in order to use matrix notation to calculate the expectation value and they noted that students often mixed up the order of the vectors and operators. Additionally, they found that students often wrote the Dirac expression $\langle\psi|\hat{A}| \psi\rangle$ as a template for whatever method they would proceed to use.

In a study by Singh, a survey administered at the beginning of a graduate quantum mechanics class had a question that asked for the expectation value of energy at a time $t$ for a wave function written as a superposition of two energy eigenstates at $t=0$ [2]. An important aspect of this question was that students were asked for the probabilities and values of an energy measurement at time $t=0$ immediately before being asked to solve for the expectation value of energy at a later time. Singh found that $67 \%$ of students answered the probabilities and values question correctly, but only $39 \%$ got the expectation value question correct. It is not reported in their paper what methods were used by students attempting to answer, other than indicating that "many students ... calculated $\langle E\rangle$ by brute-force: first writing $\langle E\rangle=\int_{-\infty}^{+\infty} \Psi^{*} \hat{H} \Psi d x$ In interviews with students, they found that many students did not know the "weighted average" definition of the expectation value (this is the method we refer to as summation). Finally, Singh found that $17 \%$ of students wrote down the Dirac expression $\langle\psi|H| \psi\rangle$ or $\langle\psi|E| \psi\rangle$ without knowing how to proceed. 
There have been several other studies that have focused on the time evolution of expectation values $[3,4]$ as well as general difficulties related to expectation values [4-6]. In this work, we focus our investigation on the methods that students use to calculate the expectation value as well as the efficacy of each method.

\section{METHODS}

This paper analyzes three years of exam questions given in quantum mechanics courses at three institutions: California State University Fullerton (CSUF) and California State Polytechnic University Pomona (CPP) are both large, public, Hispanic-serving, primarily undergraduate institutions in Southern California; and the University of Colorado (CU) is a large, public, selective, $\mathrm{PhD}$-granting institution in Boulder, Colorado. The data have been taken from quantum mechanics courses that use a "spins-first" instructional paradigm using the textbook Quantum Mechanics by David McIntyre [7]. The courses were taught by authors using interactive methods such as multiple choice concept tests, small group work, and tutorials. Expectation values are taught using both matrix and summation method at approximately the same time in all classes, with the integration method taught later in the course.

The expectation value exam questions can be classified in two different groups. The first class of questions regard systems where the state of the quantum system can be written as a wave function of position, $\psi(x)$. The second class of questions are in the context of a spin-1/2 particle where the state of the system can be written as a two-dimensional vector. Questions in the context of position asked students to solve for either the expectation value of energy or position. When the question is in the context of spin, the question usually asks for the expectation value of spin in a particular direction, for example, the expectation value of spin in the $y$-direction $\left(\left\langle S_{y}\right\rangle\right)$.

Student exam data were analyzed by the lead author for the method students used to solve for the expectation value, as well as whether or not they arrived at the correct answer. When coding for the method students used, we did not focus on whether or not the method was used correctly, but rather whether or not they tried to use the most salient feature of each method in their solution. For example, students were coded as using the integration method if they wrote an integral symbol, were coded as using matrix if they did any matrix multiplication, and were coded as summation if they added at least two terms together.

\section{DATA ANALYSIS}

Analyses are presented separately depending on the context of the question (position and spin) to account for the variation in the appropriate methods used to determine the expectation values. We have combined results from different administrations of the questions when the following criteria have been met: the questions are of the same type with the same notation used to provide the quantum state and the results from the administrations are similar.

\section{A. Expectation values in the context of position}

Exam questions in the position representation involved either a particle in an infinite square well or an electron in a hydrogen atom. Each question asked for the expectation value of energy and one of the questions also asked for the expectation value of position. In each case the question included an initial state written as a superposition of energy eigenstates. For example, a question given at CSUF asked students to consider a particle in an infinite square well with the initial state $\psi(x)=\sqrt{\frac{1}{3}} \phi_{1}(x)+i \sqrt{\frac{2}{3}} \phi_{2}(x)$ and to find the expectation value of energy and position. When solving for the expectation value of energy, it is preferable to use the summation method as the probabilities and energy eigenvalues are readily available. However, it is possible to integrate and arrive at the same answer. This requires that the Hamiltonian and energy eigenstates be written in the position representation as $H=\frac{-\hbar^{2}}{2 m} \frac{\partial^{2}}{\partial x^{2}}$ and $\phi_{n}(x)=\sqrt{\frac{2}{a}} \sin \left(\frac{n \pi x}{a}\right)$. In this example, the only suitable method for calculating $\langle\hat{x}\rangle$ is integration.

\section{Expectation value of energy}

We analyzed 129 student responses to questions asking for the expectation value of energy for a particle in either an infinite square well or hydrogen atom potential. The responses were to four different questions asked on four separate exams at two institutions (two questions per institution). As stated above, in each question the state was clearly written as a superposition of energy eigenstates and the preferred method for solving for the expectation value is summation. We found that $76 \%$ of students $(98 / 129)$ used the summation method to solve the for the expectation value. In total, $67 \%$ of students $(87 / 129)$ arrived at the correct answer using this method. There were $5 \%$ of students $(7 / 129)$ who chose to use an integral with no student arriving at the correct answer this way. This overwhelmingly supports the notion that the summation method is preferable to integration when the probabilities are readily accessible (such as when the state is written in the eigenbasis of the appropriate operator, in this case, the Hamiltonian.)

\section{Expectation value of position}

In one administration, students were also asked for the expectation value of position. The question was an additional part to the energy expectation value question discussed above, using the same wave function (which was written as a superposition of energy eigenstates). Integration is the only 
method to solve for the expectation value in this case. Of the 20 students who answered this question, 90\% (18/20) answered with an integral. Almost all students arrived at the incorrect answer (only $2(10 \%)$ answered correctly), but had a mostly correct integral expression. There were $20 \%(4 / 20)$ of students who wrote down an incorrect integral by either forgetting to include one instance of the state or the operator. No students attempted to use a summation to solve this expectation value.

\section{B. Expectation values in the context of spin}

The exam questions that asked about spin-1/2 states can be separated into two types: ones where the state is written in the eigenbasis of the operator for which they are to find the expectation value and ones where the state is written in a different basis. For example, consider a question where the state is given as $|\psi\rangle=\frac{1}{\sqrt{5}}\left(2|+\rangle_{z}-i|-\rangle_{z}\right)$, where $| \pm\rangle_{z}$ are the eigenstates of $S_{z}$. Asking for the expectation value of $S_{z}$ is of the first type, as the state is already expressed in the eigenbasis of the relevant operator. This is the "easiest" expectation value question that can be asked for this state, since it is possible to "read off" the probabilities of different measurement outcomes and use the summation method with very little algebra. It is possible to instead ask for the expectation value of $S_{y}$ (making it the second "different basis" type of expectation value question). This makes for a considerably more difficult question: since the state is not written in the relevant basis, the probabilities are no longer easily accessible and require more involved algebraic expressions to solve. We have separated our analysis for these two types of questions.

\section{Same basis}

These questions provided students with the state of a spin$1 / 2$ particle written in the $z$-basis and asked for the expectation value of $S_{z}$. There were two administrations of this type of question that are very similar, with some small differences. Both questions are reproduced below.

Administration 1: Given the following state:

$$
|\psi\rangle=\frac{1}{\sqrt{5}}\left(2|+\rangle_{z}-i|-\rangle_{z}\right) \doteq \frac{1}{\sqrt{5}}\left(\begin{array}{c}
2 \\
-i
\end{array}\right)
$$

find the expectation value of $S_{z}$.

Administration 2: Consider a spin-1/2 particle in the state

$$
|\psi(t=0)\rangle \doteq\left(\begin{array}{c}
1 / 2 \\
\sqrt{3} / 2
\end{array}\right)
$$

This particle is in a steady uniform magnetic field oriented in the $y$-direction. What is the expectation value of $S_{z}$ at $t=0$ ?
Administration 1 was on a midterm exam in a ten-week quarter-long course at CPP, while administration 2 was on a final exam in a semester-long course at CU. There are three main differences in these questions. First, in terms of the information provided, administration 1 gives the quantum state in both Dirac notation as well as matrix notation, while administration 2 only provides the state in matrix notation. Second, the particle in administration 2 is in a magnetic field. Although the question does not ask about time evolution and therefore the presence of the magnetic field does not affect the calculation, it might affect student thinking. And finally, the amount of material covered at the time of administration differs as administration 1 was on a midterm exam in a tenweek quarter-long course at CPP, while administration 2 was on a final exam in a semester-long course at CU. The percent of students using each method, along with the percent that answered correctly of those that used each method, are given in Table I. Note that the numbers may not sum to 100 as some students did not answer the question or their response did not fit into these categories.

TABLE I. Breakdown of student responses to "same basis" questions. The percent that used each method is given as well as the percent correct of those that used each method.

\begin{tabular}{l|cc|cc|cc}
\hline \hline & \multicolumn{2}{|c|}{ Summation } & \multicolumn{2}{|c|}{ Matrix } & \multicolumn{2}{c}{ Both } \\
& Used Correct & Used & Correct & Used & Correct \\
\hline Admin. 1 $(N=41)$ & $10 \%$ & $100 \%$ & $83 \%$ & $80 \%$ & $2 \%$ & $0 \%$ \\
Admin. 2 $(N=41)$ & $42 \%$ & $76 \%$ & $51 \%$ & $86 \%$ & $5 \%$ & $100 \%$ \\
\hline \hline
\end{tabular}

Comparing the choice of method between the two administrations, we find the in both administrations the matrix method was most popular, however the split was more even between matrix and summation in administration 2 . It is possible that this difference is due to question wording, but we believe it is more likely due to differences in instructional emphasis and/or the different time of the quarter/semester the exams were given. Administration 1 was given after approximately five weeks of instruction while administration 2 was given after 15 weeks. It is plausible that the additional material covered in administration 2 could have increased students understanding and facility with the summation method. Despite the differences in the number of students using each method, the efficacy of each method was similar as between $75 \%$ and $100 \%$ of students arrived at the correct answer.

\section{Different basis}

For questions that asked about a different basis, the states were presented as superpositions of $S_{z}$-eigenstates and the question asked for $\left\langle S_{y}\right\rangle$, as shown below.

Consider the state $|\psi\rangle=N\left(1|+\rangle_{z}-3 i|-\rangle_{z}\right)$.

(a) What are the possible results of a measurement of 
spin in the $y$-direction and what are the probabilities of each outcome?

(b) Find the expectation value of $S_{y}$ for this state.

Three instances of this question were asked at CSUF and $\mathrm{CU}$ on midterm exams, with a total of 93 students responding. Table II shows the percent of students who used each method and the percent of those students who got the correct answer. It was more common for students to use matrix notation, although the percent of students arriving at the correct answer was similar in each. All of the students who used both methods arrived at the correct answer. Notably, the summation method could have been straightforward using the previously calculated probabilities and values. Most of the students using the summation method who did not get the correct answer had errors when solving for the probabilities.

TABLE II. Methods used on "different basis" questions. The percent that used each method is given as well as the percent correct of those that used each method.

\begin{tabular}{c|cc|cc|cc}
\hline \hline & \multicolumn{2}{|c|}{ Summation } & \multicolumn{2}{|c|}{ Matrix } & \multicolumn{2}{c}{ Both } \\
& Used & Correct & Used & Correct & Used & Correct \\
\hline CSUF $(N=50)$ & $32 \%$ & $56 \%$ & $58 \%$ & $65 \%$ & $6 \%$ & $100 \%$ \\
CU $(N=43)$ & $33 \%$ & $64 \%$ & $51 \%$ & $73 \%$ & $14 \%$ & $100 \%$ \\
\hline All $(N=93)$ & $32 \%$ & $60 \%$ & $55 \%$ & $68 \%$ & $10 \%$ & $100 \%$ \\
\hline \hline
\end{tabular}

A different version of this question was asked on a final exam at CPP $(N=14)$ where students were asked for the expectation value of $S_{y}$. The state was provided in both Dirac notation and as a column vector. In this version of the question, students were not asked to calculate the probabilities and values beforehand, and therefore using the summation method would require additional calculations. All but one student $(93 \%)$ used the matrix method to solve and one student $(7 \%)$ used summation notation. Almost all students arrived at the correct answer.

\section{DISCUSSION}

The methods that students use to compute expectation values vary based on the question context. Students readily use the summation method for the expectation value of energy and the integration method for the expectation value of position. However, for questions asking for the expectation value of energy, the state is often written as a sum of energy eigenstates. While this allows the summation method to be used, it is possible that it is inadvertently teaching students that energy expectation values are always solved this way. It would be instructive to ask for the expectation value of energy when the state is not written in the energy eigenbasis, but rather as a simple function of $x$. For example, it is possible to ask for the expectation value of energy for the state $\psi(x)=N x(a-x)$ in an infinite square well of width $a$ (where $N$ is a normalization constant). In this case, the probabilities of different energy measurements are not easily accessible and integration is the best method for solving. We believe this question would be much more difficult for students, not only because the integration is more challenging, but because students will have to first remember what the operator of energy is, how to write it in position representation, set up the appropriate integral, and then perform the integration. We plan to ask questions of this type in future semesters.

In the context of spins, the results suggest students don't necessarily recognize or apply the summation method, even when the probabilities can be readily determined or when they have been previously calculated. We found that only $32 \%$ of students used the summation method even though they had already calculated the probabilities and values that were required. However, despite not using the method we feel was "easier", both the summation and matrix method had similar success rates. It is possible that students may simply be choosing the method they are most comfortable with. Informal conversations with students and observations in class lead us to hypothesize that the summation method requires more conceptual thought than the matrix method. The summation method requires students to recognize the possible measurement values and their probabilities before calculating the expectation value. In contrast, the matrix method can be set up and calculated without much conceptual thought. In future research we plan to perform a series of interviews to investigate whether or not students recognize multiple methods for solving the same problem, to observe if a student's preferred method for solving the expectation value changes throughout the semester, and to gain some insight into why students choose different methods.

\section{ACKNOWLEDGMENTS}

This work has been supported in part by the NSF under Grants No. DUE-1626594, 1626280, and 1626482.
[1] Gire, E., Price, E., Phys. Rev. ST Phys. Educ. Res 11, 20109 (2015)

[2] Singh, C., Am. J. Phys., 76, 227 (2008)

[3] Singh, C., Am. J. Phys., 69, 885 (2001)

[4] Zhu, G., Singh, C., Phys. Rev. ST - PER, 8, 10117 (2012)
[5] Singh, C., AIP Conference Proceedings, 818, 69 (2006)

[6] Sadaghiani, H., Bao, L., AIP Conference Proceedings, 818, 61 (2006)

[7] D. H. McIntyre, Quantum Mechanics (Pearson Education, Inc., San Francisco, CA, 2012) 